Dumitru Popa, Department of Mathematics, University of Constanta, Bd. Mamaia 127, 8700 Constanta, Romania. e-mail: dpopa@univ-ovidius.ro

\title{
ON THE VECTOR FORM OF THE LAGRANGE FORMULA, THE DARBOUX PROPERTY AND L'HÔPITAL'S RULE
}

\author{
Dedicated to the memory of my colleague Vasile Ene.
}

\begin{abstract}
We prove that the well-known Lagrange formula, the Darboux property and a classical result concerning the connected graph of a differentiable function are specific for $\mathbb{R}$, and surprisingly, the rule of L'Hôpital is also true for the vector case.
\end{abstract}

Proposition 1. Let $X$ be a topological vector space. Then the following assertions are equivalent:

1. For each $f:[0,1] \rightarrow X, f$ continuous on $[0,1]$ and differentiable on $(0,1)$, there exists $c \in(0,1)$ such that: $f(1)-f(0)=f^{\prime}(c)$.

2. For each $f:[0,1] \rightarrow X, f$ continuous on $[0,1]$ and differentiable on $(0,1)$ with $f(1)=f(0)$, there exists $c \in(0,1)$ such that: $f^{\prime}(c)=O$.

3. $X$ is a real topological vector space and $\operatorname{dim}_{\mathbb{R}} X=1$.

Proof. i) or ii) $\Rightarrow$ iii) Let $x \in X, x \neq O$. Let us suppose that $X$ is a complex topological vector space. In this case, let

$$
f:[0,1] \rightarrow X, \quad f(t)=(\cos 2 \pi t+i \sin 2 \pi t) x .
$$

The hypotheses from i) or ii) are satisfied, thus there exists $c \in(0,1)$ such that: $f^{\prime}(c)=O$ (because $\left.f(1)=f(0)=x\right)$. But

$$
\left.f^{\prime}(c)=2 \pi(-\sin 2 \pi c+i \cos 2 \pi c)\right) x,
$$

Key Words: Lagrange Formula, Darboux Property, L'Hôpital's Rule

Mathematical Reviews subject classification: 26A24

Received by the editors January 16, 1998 
so $-\sin 2 \pi c+i \cos 2 \pi c=0$, which contradicts the fact that $\cos ^{2} 2 \pi c+\sin ^{2} 2 \pi c=1$. Hence $X$ is a real topological vector space. In this case, let $y \in X$ and $g:[0,1] \rightarrow X, g(t)=x \cos 2 \pi t+y \sin 2 \pi t$. From i) or ii), there exists $c \in(0,1)$ such that $g^{\prime}(c)=O$, hence $x \sin 2 \pi c=y \cos 2 \pi c$. If $\cos 2 \pi c=0$, then $\sin 2 \pi c= \pm 1$. It follows that $O=y \cdot \cos 2 \pi c= \pm x$, so $x=O$, which is impossible. Thus $\cos 2 \pi c \neq 0$ and $y=(\tan 2 \pi c) x$, hence $\operatorname{dim}_{\mathbb{R}} X=1$.

iii) $\Rightarrow$ i) or ii) Let $X$ be a topological real vector space, $\operatorname{dim}_{\mathbb{R}} X=1$, and let $f:[0,1] \rightarrow X$ be as in i) or ii). Let $x \in X, x \neq O$. Since $f(t) \in X$, there exists an unique $\varphi(t) \in \mathbb{R}$ such that $f(t)=\varphi(t) x, \forall t \in[0,1]$. It is easy to prove that $\varphi$ is continuous on $[0,1]$ and differentiable on $(0,1)$. Then the classical Rolle or Lagrange theorems applied to $\varphi:[0,1] \rightarrow \mathbb{R}$ imply i) or ii).

Proposition 2. Let $X$ be a normed space. Then the following assertions are equivalent:

1. For each function $f:[0,1) \rightarrow X$ differentiable on $[0,1)$, it follows that: $f^{\prime}([0,1)) \subset X$ is a connected subset.

2. $X$ is a real normed space and $\operatorname{dim}_{\mathbb{R}} X=1$.

Proof. i) $\Rightarrow$ ii) Let $x \in X$, with $\|x\|=1$. Suppose that $X$ is a complex normed space. Let $f:[0,1) \rightarrow X$,

$$
f(t)= \begin{cases}0 & \text { if } t=0 \\ \left(t^{2} \sin \frac{1}{t}+i t^{2} \cos \frac{1}{t}\right) x & \text { if } t \in(0,1)\end{cases}
$$

Obviously, $f$ is differentiable on $(0,1)$. For $t>0$,

$$
\left\|\frac{f(t)-f(0)}{t}\right\|=\left\|\left(t \sin \frac{1}{t}+i t \cos \frac{1}{t}\right) x\right\|=t .
$$

Thus

$$
f^{\prime}(t)=\left\{\begin{array}{ll}
0 & \text { if } t=0 \\
{\left[2 t \sin \frac{1}{t}-\cos \frac{1}{t}+i\left(2 t \cos \frac{1}{t}+\sin \frac{1}{t}\right)\right] x} & \text { if } t \in(0,1)
\end{array} .\right.
$$

Since $f^{\prime}([0,1)) \subset X$ is a connected subset then :

$$
\inf _{0<t<1}\left\|\left(2 t \sin \frac{1}{t}-\cos \frac{1}{t}\right)+i\left(2 t \cos \frac{1}{t}+\sin \frac{1}{t}\right) x\right\|=0,
$$

or $\|x\|=1, \inf _{0<t<1}\left(4 t^{2}+1\right)=0$, a contradiction. Thus $X$ is a real normed space. Let $y \in X$ and let $F:[0,1) \rightarrow X$ be defined as follows:

$$
F(t)=\left\{\begin{array}{ll}
0 & \text { if } t=0 \\
\left(t^{2} \sin \frac{1}{t}\right) x+\left(t^{2} \cos \frac{1}{t}\right) y & \text { if } t \in(0,1)
\end{array} .\right.
$$


Clearly $F$ is differentiable and

$$
F^{\prime}(t)= \begin{cases}0 & \text { if } t=0 \\ x\left(2 t \sin \frac{1}{t}-\cos \frac{1}{t}\right)+y\left(2 t \cos \frac{1}{t}+\sin \frac{1}{t}\right) & \text { if } t \in(0,1)\end{cases}
$$

By i), it follows that $F^{\prime}([0,1)) \subset X$ is connected, hence

$$
\inf _{0<t<1}\|x h(t)+y g(t)\|=0,
$$

where

$$
h(t)=2 t \sin \frac{1}{t}-\cos \frac{1}{t}, \quad g(t)=2 t \cos \frac{1}{t}+\sin \frac{1}{t}, \quad t \in(0,1] .
$$

By (1), there exists a sequence $\left(t_{n}\right)_{n \in N} \subset(0,1)$ such that

$$
\left\|x h\left(t_{n}\right)+y g\left(t_{n}\right)\right\| \rightarrow 0 .
$$

The sequence $\left(t_{n}\right)_{n \in N}$ being bounded, we can choose a subsequence (which for simplicity will also be denoted by $\left.\left(t_{n}\right)_{n \in N}\right)$ convergent to $t_{o} \in[0,1]$. If $0<t_{0} \leq 1$, using the continuity of the functions $h$ and $g$ on $(0,1)$, by (2), it follows that $\left\|x h\left(t_{0}\right)+y g\left(t_{0}\right)\right\|=0$, hence $x h\left(t_{0}\right)+y g\left(t_{0}\right)=O$. If $g\left(t_{0}\right)=O$, as $h^{2}\left(t_{0}\right)+g^{2}\left(t_{0}\right)=4 t_{0}^{2}+1>1$, we obtain: $x h\left(t_{0}\right)=-y g\left(t_{0}\right)=0$. Then, $h\left(t_{0}\right) \neq 0$, implies $x=0$, which contradicts the fact that $\|x\|=1$. Thus $g\left(t_{0}\right) \neq O$ and $y=\lambda x$, with $\lambda=-\frac{h\left(t_{0}\right)}{g\left(t_{0}\right)} \in \mathbb{R}$. If $t_{0}=0$, then $t_{n} \rightarrow 0$, and by (2) we have:

$$
\begin{gathered}
\left\|y \sin \frac{1}{t_{n}}-x \cos \frac{1}{t_{n}}\right\| \leq \\
\leq\left\|x h\left(t_{n}\right)+y g\left(t_{n}\right)\right\|+\left\|2 t_{n} \sin \frac{1}{t_{n}} x\right\|+\left\|2 t_{n} \cos \frac{1}{t_{n}} y\right\| \leq \\
\leq 2\left|t_{n}\right|(\|x\|+\|y\|)+\left\|x h\left(t_{n}\right)+y g\left(t_{n}\right)\right\| \rightarrow 0 .
\end{gathered}
$$

Thus:

$$
\left\|y \sin \frac{1}{t_{n}}-x \cos \frac{1}{t_{n}}\right\| \rightarrow 0 .
$$

From here we obtain:

$$
\inf _{0<t<1}\left\|y \sin \frac{1}{t}-x \cos \frac{1}{t}\right\| \leq\left\|y \sin \frac{1}{t_{n}}-x \cos \frac{1}{t_{n}}\right\| \rightarrow 0,
$$

i.e.

$$
\inf _{0<t<1}\left\|y \sin \frac{1}{t}-x \cos \frac{1}{t}\right\|=0
$$


or using the periodicity of $\sin e$ and $\cos e$,

$$
\inf _{0 \leq \theta \leq 2 \pi}\|y \sin \theta-x \cos \theta\|=0 .
$$

Let $0 \leq \theta_{n} \leq 2 \pi$ be such that: $\left\|y \sin \theta_{n}-x \cos \theta_{n}\right\| \rightarrow 0$. Extract a convergent subsequence which will be denoted also by $\theta_{n} \rightarrow \theta$, where $\theta \in[0,2 \pi]$. Hence: $\|y \sin \theta-x \cos \theta\|=0$, i.e. $y \sin \theta=x \cos \theta$. If $\sin \theta=0$, then $\cos \theta= \pm 1$, from where $0=y \sin \theta=x \cos \theta= \pm x$, i.e. $x=0$, contradiction! Thus, $\sin \theta \neq 0$ and hence: $y=\lambda x$, with $\lambda=\cot \theta$, i.e. ii) is proved.

ii) $\Rightarrow$ i) Let $X$ be a normed space with $\operatorname{dim}_{\mathbb{R}} X=1, x \in X$ with $\|x\|=1$, and let $f:[0,1) \rightarrow X$ be a differentiable function. Then there exists a unique element $\varphi(t) \in \mathbb{R}$ such that $f(t)=\varphi(t) \cdot x, \forall t \in[0,1)$, i.e. there exists $\varphi:[0,1) \rightarrow \mathbb{R}$ such that $f(t)=\varphi(t) x$. Because $f$ is differentiable on $[0,1)$ and $\|x\|=1$ we obtain easily that $\varphi$ is derivable on $[0,1)$. Using the Darboux theorem for $\varphi:[0,1) \rightarrow \mathbb{R}$, we obtain that $I=\varphi^{\prime}([0,1)) \subset \mathbb{R}$ is an interval, i.e. a connected set. Since $f^{\prime}(t)=\varphi^{\prime}(t) x, \forall t \in[0,1)$, it follows that $f^{\prime}([0,1))=x \cdot I$ is a connected subset in $X$.

Recall that if $X$ is a topological space a subset $A \subset X$ is called path connected if for each $x_{0}, x_{1} \in A$, there exists $\gamma:[0,1] \rightarrow X$, a continuous functions such that: $\gamma(0)=x_{0}, \gamma(1)=x_{1}, \operatorname{Im} \gamma \subset A$. In the sequel we need the following, probably well-known result. We give the proof for the completeness.

Proposition 3. Let $X$ be a metric space, $f:(0, \infty) \rightarrow X$, be a continuous function, $a \in X$ and $A=\{(0, a)\} \cup\{(x, f(x)) \mid x>0\} \subset(0, \infty) \times X$. Then:

1. $A$ is connected if and only if there exists a sequence $\left(x_{n}\right)_{n \in N} \subset(0, \infty)$ such that: $x_{n} \rightarrow 0$, and $f\left(x_{n}\right) \rightarrow a$.

2. A is path connected if and only if: $\lim _{x \rightarrow 0, x>0} f(x)=a$.

Proof. a) If $A$ is connected then: $d((0, a), G)=\inf _{x>0} d((0, a),(x, f(x)))=0$, $\inf _{x>0}(x+d(f(x), a))=0$. ( Here $G$ is the graph of $f$ ). Conversely, this condition implies: $(0, a) \in \bar{G},\{(0, a)\} \cap \bar{G}=\{(0, a)\} \neq \emptyset$, hence: $A=\{(0, a)\} \cup G$ is connected. But $\inf _{x>0}(x+d(f(x), a))=0$, if and only if there exists a sequence $\left(x_{n}\right)_{n \in N} \subset(0, \infty)$ such that: $x_{n} \rightarrow 0$, and $f\left(x_{n}\right) \rightarrow a$.

b) Let us suppose that $A$ is path connected. Then for the points $(0, a)$ and $(1, f(1)) \in A$ there exists a continuous path contained in $A$ i.e. $\gamma:[0,1] \rightarrow \mathbb{R}^{2}$, $\gamma=(g, h)$, continuous such that: $\gamma(0)=(0, a), \gamma(1)=(1, f(1)), \operatorname{Im} \gamma \subset A$. So: $g(0)=0, g(1)=1$. Let

$$
E=\{t \in[0,1] \mid g(t)=0\}=g^{-1}(\{0\}),
$$


$\alpha=\sup E$. The set $E$ is nonvoid, since $g(0)=0$. As $g(1)=1>0, \alpha \neq 1$, i.e. $0 \leq \alpha<1$. But $g$ is continuous and $E$ is closed, so $\alpha \in E, g(\alpha)=0$, i.e. $\alpha=\max E$. Hence if $t>\alpha, t \in[0,1], g(t) \neq 0$, so $\gamma(t)=(g(t), h(t)) \in A$, i.e. $g(t)>0, h(t)=f(g(t))$. Thus: $\forall t \in(\alpha, 1], h(t)=f(g(t))$ and using the continuity of $h$ :

$$
\lim _{t \rightarrow \alpha, t>\alpha} h(t)=h(\alpha), \quad \lim _{t \rightarrow \alpha, t>\alpha} f(g(t))=h(\alpha) .
$$

We prove that:

$$
\lim _{x \rightarrow 0, x>0} f(x)=h(\alpha) .
$$

If this is not so, then: there exists $\varepsilon_{0}>0$, a sequence $0<x_{n}<\frac{1}{n}$, such that:

$$
d\left(f\left(x_{n}\right), h(\alpha)\right) \geq \varepsilon_{0}, \quad \forall n \in N .
$$

From $g(\alpha)=0<x_{n}<1=g(1)$, and the Darboux property of $g$, there is $\alpha<t_{n}<1$, such that: $g\left(t_{n}\right)=x_{n}$. Extract a convergent subsequence: $t_{k_{n}} \rightarrow t \in[\alpha, 1]$. The continuity of $g$ implies: $x_{k_{n}}=g\left(t_{k_{n}}\right) \rightarrow g(t), g(t)=0$, $t \in E, t \leq \alpha$. But $t \geq \alpha$, so $t=\alpha$. Hence: $t_{k_{n}} \rightarrow \alpha, t_{k_{n}}>\alpha$ and (3) gives: $\left.f\left(g\left(t_{k_{n}}\right)\right) \rightarrow h(\alpha), f\left(x_{k_{n}}\right)\right) \rightarrow h(\alpha)$, which contradicts (5). Thus (4) is proved. But if $A$ is path connected, then it is connected, so by a) there exists a sequence $\left(x_{n}\right)_{n \in N} \subset(0, \infty)$ such that: $x_{n} \rightarrow 0$, and $f\left(x_{n}\right) \rightarrow a$. Using (4):

$$
f\left(x_{n}\right) \rightarrow h(\alpha), \quad h(\alpha)=a, \text { i.e. } \lim _{x \rightarrow 0, x>0} f(x)=a .
$$

The converse is clear.

Using the same functions as in the Proposition 2, with the help of Proposition 3 , we must show that a classical result, see [3], is also specified to $\mathbb{R}$.

Proposition 4. Let $X$ be a normed space. Then the following assertions are equivalent:

1. For each function $f:[0,1) \rightarrow X$ differentiable on $[0,1)$, it follows that the graph of $f^{\prime}$ is a connected subset.

2. $X$ is a real normed space and $\operatorname{dim}_{\mathbb{R}} X=1$.

Also, minor changes in the above proofs, show that, they are also true if instead of a normed space $X$, we suppose that $X$ is a vector space endowed with a $F$-norm, or a $p$-norm $(0<p \leq 1)$, see [2] for definitions.

Now a positive result for the classical L'Hôpital rule.

Theorem 1. (The L'Hôpital rule, cases $\left[\frac{0}{0}\right]$, resp. $[-\bar{\infty}]$ )

Let $X$ be a normed space, $(a, b) \subseteq \mathbb{R}$ be an open interval and $f:(a, b) \rightarrow X$, $g:(a, b) \rightarrow \mathbb{R}$ two functions with the properties: 
1. $\lim _{\substack{t \rightarrow a \\ t>a}} f(t)=0$ and $\lim _{\substack{t \rightarrow a \\ t>a}} g(t)=0$; (respectively $\left.\lim _{\substack{t \rightarrow a \\ t>a}}|g(t)|=\infty\right)$;

2. $f$ is differentiable on $(a, b)$ and $g$ is differentiable on $(a, b)$;

3. $g^{\prime}(t) \neq 0, \forall t \in(a, b)$;

4. The limit $\lim _{\substack{t \rightarrow a \\ t>a}} \frac{f^{\prime}(t)}{g^{\prime}(t)} \in X$ exists.

Then there exists

$$
\lim _{\substack{t \rightarrow a \\ t>a}} \frac{f(t)}{g(t)} \in X \quad \text { and in addition: } \lim _{\substack{t \rightarrow a \\ t>a}} \frac{f(t)}{g(t)}=\lim _{\substack{t \rightarrow a \\ t>a}} \frac{f^{\prime}(t)}{g^{\prime}(t)} .
$$

Proof. Since $g^{\prime}$ has the Darboux property, by 3) and 2), it follows that $g^{\prime}$ has a constant sign on $(a, b)$, hence $g$ is strictly monotone on $(a, b)$. Let

$$
x=\lim _{\substack{t \rightarrow a \\ t>a}} \frac{f^{\prime}(t)}{g^{\prime}(t)} \in X .
$$

Then for $\varepsilon>0$, there is a $\delta>0$ such that:

$$
\left\|\frac{f^{\prime}(t)}{g^{\prime}(t)}-x\right\|<\varepsilon, \text { hence: }\left\|f^{\prime}(t)-x g^{\prime}(t)\right\|<\varepsilon\left|g^{\prime}(t)\right|,
$$

on each compact subinterval $[u, v] \subset(a, a+\delta)$. By the Denjoy-Bourbaki Theorem, it follows that:

$$
\|f(v)-x g(v)-f(u)+x g(u)\| \leq \varepsilon|g(v)-g(u)| .
$$

We prove the case $\left[\frac{0}{0}\right]$. By 1$)$ and $(*)$, for $u \searrow a$ it follows that:

$$
\|f(v)-x g(v)\| \leq \varepsilon|g(v)| \text {, hence: }\left\|\frac{f(v)}{g(v)}-x\right\|<\varepsilon .
$$

Therefore

$$
\lim _{\substack{v \rightarrow a \\ v>a}} \frac{f(v)}{g(v)}=x .
$$

We prove the case $[\bar{\infty}]$. We may suppose that $g(t) \neq 0$ on $[u, v]$. By 1$)$ and $(*)$, we have:

$$
\left\|\frac{f(u)}{g(u)}-x\right\| \leq \frac{\|f(v)-x g(v)\|}{|g(u)|}+\epsilon\left|\frac{g(v)}{g(u)}-1\right|,
$$


hence :

$$
\limsup _{u \rightarrow a, u>0}\left\|\frac{f(u)}{g(u)}-x\right\| \leq \epsilon .
$$

Since $\varepsilon$ is arbitrary, we obtain that:

$$
x=\lim _{\substack{u \rightarrow a \\ u>a}} \frac{f(u)}{g(u)} .
$$

\section{References}

[1] N. Bourbaki, Fonctions d'une variable reele, Paris, Herman, 1961.

[2] N. J. Kalton, N. T. Peck, J. W. Roberts, An F-space sampler, Cambridge Univ. Press, 1984.

[3] K. Kuratowski, Topology, vol. II, Academic Press, 1968. 
Dumitru Popa 